\title{
Atomic structure evolution in bulk metallic glass under compressive stress
}

G. Wang, N. Mattern, S. Pauly, J. Bednarčik, and J. Eckert

Citation: Appl. Phys. Lett. 95, 251906 (2009); doi: 10.1063/1.3276274

View online: https://doi.org/10.1063/1.3276274

View Table of Contents: http://aip.scitation.org/toc/apl/95/25

Published by the American Institute of Physics

\section{Articles you may be interested in}

Origin of yielding in metallic glass: Stress-induced flow

Applied Physics Letters 104, 251901 (2014); 10.1063/1.4884066

Structural features of plastic deformation in bulk metallic glasses

Applied Physics Letters 106, 031903 (2015); 10.1063/1.4906305

Plasticity-improved $\mathrm{Zr}$-Cu-Al bulk metallic glass matrix composites containing martensite phase

Applied Physics Letters 87, 051905 (2005); 10.1063/1.2006218

Bulk metallic glass formation in the binary $\mathrm{Cu}-\mathrm{Zr}$ system

Applied Physics Letters 84, 4029 (2004); 10.1063/1.1751219

Thickness of shear bands in metallic glasses

Applied Physics Letters 89, 071907 (2006); 10.1063/1.2336598

Reversible transition of deformation mode by structural rejuvenation and relaxation in bulk metallic glass Applied Physics Letters 101, 121914 (2012); 10.1063/1.4753998

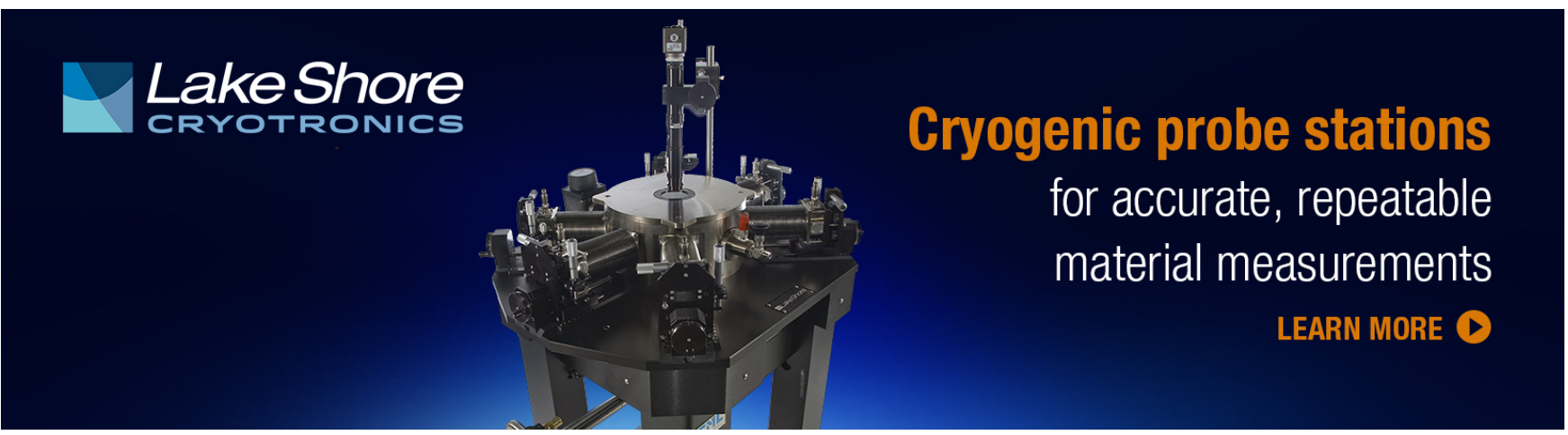




\title{
Atomic structure evolution in bulk metallic glass under compressive stress
}

\author{
G. Wang, ${ }^{1, a)}$ N. Mattern, ${ }^{1}$ S. Pauly, ${ }^{1}$ J. Bednarčik, ${ }^{2}$ and J. Eckert ${ }^{1,3}$ \\ ${ }^{1}$ Institute for Complex Materials, IFW-dresden, Helmholtzstr. 20, D-01069 Dresden, Germany \\ ${ }^{2}$ HASYLAB-DESY, Notkestr. 85, D-22603 Hamburg, Germany \\ ${ }^{3}$ Institute of Materials Science, TU Dresden, D-01062 Dresden, Germany
}

(Received 9 October 2009; accepted 1 December 2009; published online 23 December 2009)

\begin{abstract}
The structural behavior of $\mathrm{Cu}_{64.5} \mathrm{Zr}_{35.5}$ bulk metallic glass under compressive stress was investigated by means of in situ high energy x-ray synchrotron diffraction. The topological and chemical short-range order of the glass changes reversible upon loading within the elastic range. The number density of $\mathrm{Cu}-(\mathrm{Zr}, \mathrm{Cu})$ and $\mathrm{Zr}-\mathrm{Zr}$ nearest neighbor atomic pairs becomes oriented along the loading direction. The macroscopic stress state is reflected by the medium-range order. The determination of the components of the strain tensor from the shift of the positions of the nearest neighbor distances is not possible due to the structure changes. (C) 2009 American Institute of Physics.
\end{abstract}

[doi:10.1063/1.3276274]

X-ray diffraction (XRD) or neutron diffraction are well established methods to measure the elastic strain in crystalline materials. ${ }^{1}$ Using high energy $\mathrm{x}$-ray synchrotron radiation, Poulsen $e t a .^{2}$ demonstrated the possibility to estimate the components of the strain tensor in a bulk metallic glass (BMG). Further in situ studies under applied stress for several, mostly Zr-based BMGs focused on the global strain state and the elastic moduli. ${ }^{3-7}$ The general conclusion of these studies is that the shift of interatomic distances $r_{\mathrm{i}}$ between 5-20 A representing the strain of the medium-range order (MRO) is consistent with macroscopic elastic deformation of the glass. The nearest-neighbor shell at $r_{\mathrm{i}}<4 \AA$ exhibits a different behavior and applying formally the data analysis results then in different elastic moduli. ${ }^{2,3}$ As an explanation the possible dependence on the atomic environment as well as the specific atom pairs were discussed. ${ }^{3}$ On the other side, Suzuki and Egami ${ }^{8}$ demonstrated that relaxation, elastic or plastic deformation is related to complex atomic rearrangement. For binary $\mathrm{Cu}-\mathrm{Zr}$ glassy ribbons obtained by melt spinning recently, changes in the short-range order (SRO) under tensile stress were observed well below the elastic limit. ${ }^{8}$

In this letter, we analyze the changes in SRO of a $\mathrm{Cu}_{64.5} \mathrm{Zr}_{35.5}$ BMG under compressive stress. The partial contributions to the first near-neighbor shell of the binary glassy alloy are analyzed in detail, which give evidence of directionally dependent structural changes under applied stress.

Alloy ingots with nominal composition of $\mathrm{Cu}_{64.5} \mathrm{Zr}_{35.5}$ were prepared by arc melting a mixture of the pure elements (purities of $99.99 \%$ or higher), followed by suction casting into a $\mathrm{Cu}$-mold to form rodlike BMGs with a size of $\Phi 1$ $\times 30 \mathrm{~mm}$. The compression samples with geometry $\Phi 1$ $\times 2 \mathrm{~mm}^{2}$ were cut from the rods. Macroscopic compression tests were carried out with an Instron 8562-type machine at an initial strain rate of $2 \times 10^{-4} \mathrm{~s}^{-1}$. Three samples were measured to ensure reproducible results. In situ XRD was performed under compression at the BW5 beam-line at HASYLAB (DESY Hamburg, Germany) using a straining system (Kammrath and Weiss $\mathrm{GmbH}$ ). The load was stepwise increased up to $1200 \mathrm{~N}$ in steps of $200 \mathrm{~N}$ and then further

${ }^{a)}$ Electronic mail: g.wang@ifw-dresden.de. increased up to $1450 \mathrm{~N}$ (fracture load) with decreasing step sizes of 100,50 , and $10 \mathrm{~N}$. The experimental details were already documented in Ref. 5.

The nominal stress-strain curve under compressive load of the as-cast glass shows an elastic deformation up to $1800 \pm 20 \mathrm{MPa}$ followed by a yielding behavior without significant plastic flow before fracture (Fig. 1). The elastic modulus measured from this macroscopic compression test is $100 \pm 3 \mathrm{GPa}$. The inset of Fig. 1 shows the diffraction pattern of the $\mathrm{Cu}_{64.5} \mathrm{Zr}_{35.5}$ BMG without applied stress, exhibiting diffuse rings representing its glassy nature. Upon compression loading, the diffraction rings become elliptical, which can be characterized by intensity curves of $I(q, \chi)$ with respect to the polar coordinates $(q, \chi)$.

The atomic structure of glassy materials is usually isotropic. However, this situation is no more valid in the presence of stress. The calculation of the atomic pair correlation function (PDF) using the usual sine-Fourier transformation of the structure factor for anisotropic glass may result in systematic errors. ${ }^{9}$ In this case, the directionally dependent PDF and analogously the structure factor can be expressed by expansion into spherical harmonics. ${ }^{10,11}$ As such, the structure factor and the PDF can be separated into two components, i.e., isotropic structure factor, $S_{0}(q)$, and isotropic PDF, $\rho_{0}(r)$, and anisotropic components of structure factor, $S_{l}(q)$ and PDF, $\rho_{l}(r)$, respectively.

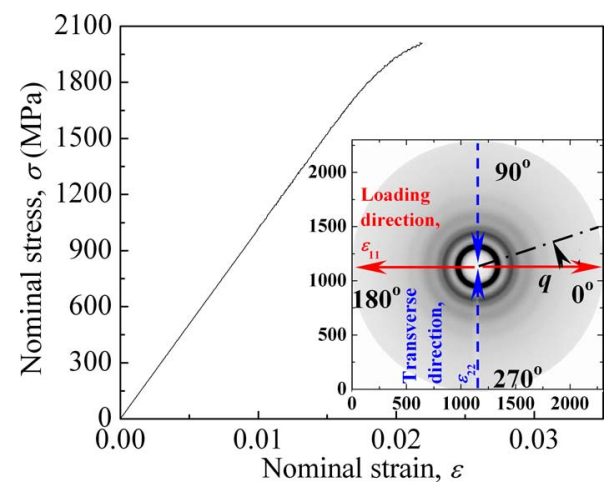

FIG. 1. (Color online) Compressive nominal stress-strain curves of the ascast $\mathrm{Cu}_{64.5} \mathrm{Zr}_{35.5}$ BMG. Inset shows a two-dimensional diffraction pattern of the $\mathrm{Cu}_{64.5} \mathrm{Zr}_{35.5}$ BMG without applied stress. 


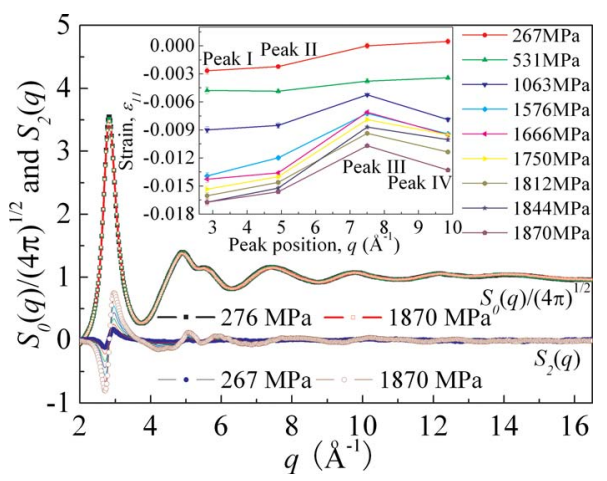

FIG. 2. (Color online) Isotropic component, $S_{0}(q)$, and anisotropic component, $S_{2}(q)$, of the structure factor at different stresses. Inset shows the strain values measured from four maxima shifting in the structure factor at different stresses in the loading direction.

Neglecting order terms $l>2$, the components can be calculated from the structure factors in the loading and transverse directions ${ }^{8,9}$

$$
\begin{aligned}
& S_{0}(q)=\frac{\sqrt{4 \pi}}{3}\left[S\left(q, \chi=0^{\circ}\right)+2 S\left(q, \chi=90^{\circ}\right)\right], \\
& S_{2}(q)=\sqrt{\frac{16 \pi}{45}}\left[S\left(q, \chi=0^{\circ}\right)-S\left(q, \chi=90^{\circ}\right)\right] .
\end{aligned}
$$

The components of $\rho_{l}(r)$ are then related by a transformation

$$
\rho_{l}(r)=\frac{(i)^{l}}{2 \pi^{2}} \int S_{l} J_{l}(q r) q^{2} d q,
$$

where $J_{l}$ is the spherical Bessel function with $J_{0}(q r)=\sin (q r) / q r, \quad$ and $\quad J_{2}(q r)=\left[3 /(q r)^{3}-1 / q r\right] \sin (q r)$ $-3 /(q r)^{2} \cos (q r)$.

The atomic density of any direction, $\chi$, is then given by

$$
\rho(r, \chi)=\rho_{0}(r) \sqrt{\frac{1}{4 \pi}}+\rho_{2}(r) \sqrt{\frac{5}{16 \pi}}\left(3 \cos \chi^{2}-1\right) .
$$

Figure 2 compares the calculated isotropic structure factors, $S_{0}(q)$, of the as-cast BMG at $\sigma=267$ and $1870 \mathrm{MPa}$. The curves are identical within the error limits. The anisotropic part of the structure factor, $S_{2}(q)$, increases gradually as a function of stress. The position of the first maxima in $S(q, \chi)$ shifts linearly with stress to higher $q$-values for the loading direction, and to lower $q$-values for the transverse direction, respectively. The calculated strain values $\varepsilon(\sigma, \chi)$ $=\left[q_{1}(\sigma, \chi)-q_{1}(0, \chi)\right] / q_{1}(\sigma, \chi)$ (inset of Fig. 2) are within the error limits in agreement with the macroscopic strain. An analysis of the shift in the second and higher maxima results in different, reduced values of the calculated strain also given by the inset of Fig. 2, which points to the limitation of the applied method for glassy materials. The necessary condition that all interatomic distances are proportionally changed under stress is obviously not valid and this is an indication of structural changes in the elastic range.

Figure 3(a) shows the isotropic component of the PDF, $\rho_{0}(r)$, for two directions as well as the anisotropic component, $\rho_{2}(r)$. The $\rho_{0}(r)_{1870 \mathrm{MPa}}$ and $\rho_{0}(r)_{267 \mathrm{MPa}}$ curves are almost identical. The amplitude of the maxima of $\rho_{2}(r)$ in-
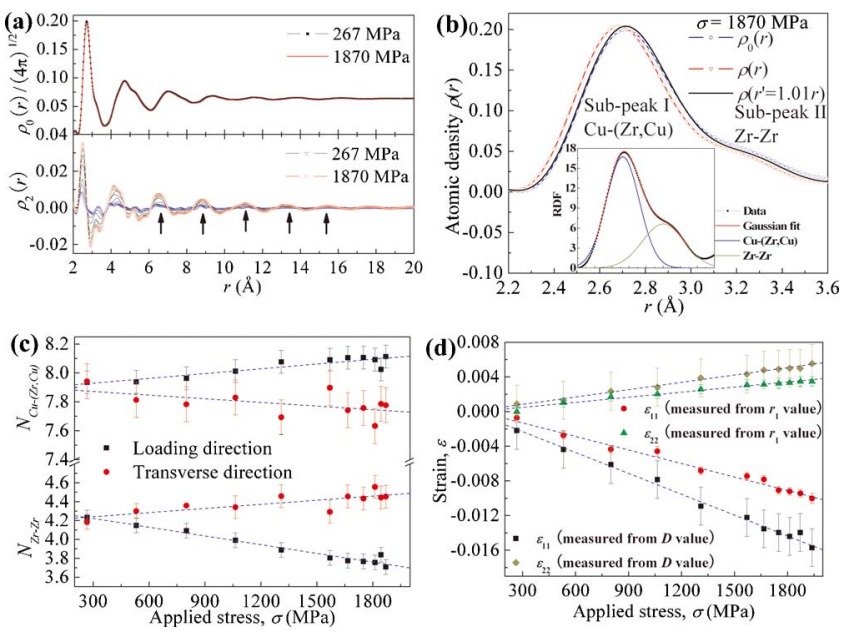

FIG. 3. (Color online) Results from the PDF analysis: (a) Isotropic component, $\rho_{0}(r)$, and anisotropic component, $\rho_{2}(r)$, of the pair correlation function. (b) Changes in the first maximum of atomic density, $\rho(r)$, at $1870 \mathrm{MPa}$ in the loading direction. Inset shows deconvolution of the first nearestneighbor shell into two Gaussians for the BMG compressed at $267 \mathrm{MPa}$ in the loading direction. (c) Coordination numbers for the $\mathrm{Cu}-(\mathrm{Zr}, \mathrm{Cu})$ and $\mathrm{Zr}-\mathrm{Zr}$ atomic pairs as functions of the applied stress. (d) Strain values vs applied stress of the BMG measured from the first maximum in the PDF and from the $D$ values.

creases with stress, indicating that anisotropy of the structure becomes obviously up to about $16 \AA$ as pointed out by arrows in Fig. 3(a).

Figure 3(b) demonstrates the change in the SRO of the $\mathrm{Cu}_{64.5} \mathrm{Zr}_{35.5}$ glass under applied stress by comparing the first maximum of the PDF of the unstrained state with that under $1870 \mathrm{MPa}$ compressive stress. The two maxima in the first nearest-neighbor shell can be attributed to the $\mathrm{Cu}-\mathrm{Cu}$ plus $\mathrm{Zr}-\mathrm{Cu}$ (Subpeak I at $r=2.7 \AA$ ), and to the $\mathrm{Zr}-\mathrm{Zr}$ (Subpeak II at $r=3.2 \AA$ ) pairs, respectively. Beside the shift of the maxima positions the intensities of the two subpeaks change with stress. Also shown in Fig. 3(b) is the PDF curve, $\rho\left(r^{\prime}\right)$, of the stressed state after normalization the maxima position by $r^{\prime}=r / 1.01$. In the loading direction the intensity of the Subpeak I increases, and decreases for the Subpeak II, respectively. For the transverse direction change becomes reversed as it follows the $\chi$-dependence of the anisotropic component $\rho_{2}(r)$ contribution to the PDF in Eq. (4). The integrations over the two components of the first maximum of the radial distribution function, $4 \pi r^{2} \rho(r)$, are related to the atomic nearest-neighbor numbers. The averaged weighted coordination numbers calculated by a fit of two Gaussian curves [inset of Fig. 3(b)] are plotted in Fig. 3(c) as a function of the stress. These values are not identical with partial coordination number, $N_{i j}^{1}$, estimated only under a presumption that individual atomic pairs are resolved in the total PDF curve, which is not the case. Due to the systematic errors in experimental data and data evaluation, the accuracy of coordination numbers is usually $\pm 10 \%$. Here we are focusing on the relative change of nearest-neighbor numbers which can be determined much more precisely. Because the isotropic part is not altered with stress, the changes are related to the anisotropic part of $\rho_{2}(r)$ only. For the $\mathrm{Cu}-(\mathrm{Zr}, \mathrm{Cu})$ pairs, the coordination number increases in the loading direction but decreases in the transverse direction and an opposite behavior occurs for the $\mathrm{Zr}-\mathrm{Zr}$ pairs. The coordination number changes reflect the topological rearrangements within the 
atomic nearest-neighbor environment in the elastic stress state. The total coordination numbers of the $\mathrm{Cu}-(\mathrm{Zr}, \mathrm{Cu})$ pair and the $\mathrm{Zr}-\mathrm{Zr}$ pair, i.e., $\left(N_{\mathrm{Cu}-(\mathrm{Zr}, \mathrm{Cu})}^{\text {loading }}+N_{\mathrm{Cu}-(\mathrm{Zr}, \mathrm{Cu})}^{\mathrm{transvere}}\right) / 2$ and $\left(N_{\mathrm{Zr}-\mathrm{Zr}}^{\text {loading }}+N_{\mathrm{Zr}-\mathrm{Zr}}^{\mathrm{transvers}}\right) / 2$, remain almost constant at $7.9 \pm 0.1$ and $4.2 \pm 0.2$, respectively, which suggests that, for the $\mathrm{Zr}-\mathrm{Zr}$ pair, the bond reorientation is processed by breaking the bonds in the loading direction and reforming new bonds in the transverse direction and the opposite behavior occurs in the $\mathrm{Cu}-(\mathrm{Zr}, \mathrm{Cu})$ pair. The observed structural changes in the elastic range are completely reversible. The PDF of BMGs after unloading from yield stress did not show significant difference to the as-cast state. Due to the atomic rearrangement, also the nearest-neighbor distances are not only influenced by the stress. Calculations of strain from the shift in the first maximum positions in the PDF are therefore not in agreement with macroscopic values.

The behavior of the PDF at larger $r$-values representing the MRO of metallic glasses can be well approximated by a damped oscillation ${ }^{12,13}$

$$
\rho(r)-1=\frac{A}{r^{\beta}} \exp \left(-\frac{r}{\xi}\right) \sin \left(\frac{2 \pi r}{D}+\Phi\right),
$$

where $A$ is a normalization constant (in the present study, the value of $A$ is 6.2.), $\beta$ is an exponent $(\sim 0.69),{ }^{13} \xi$ is a decay exponent, $D$ is the oscillation frequency, and $\Phi$ is the phase shift constant. The parameters $D$ and $\xi$ were determined by a fit of Eq. (5) to the PDFs for $r>6.3 \AA$. The parameter $D$ is related to the maxima positions of the PDF. Then the relative shift of the $D$ value reflects the averaged strain value in the MRO, i.e., $\varepsilon_{x}=[D(\sigma, x)-D(0, x)] / D(\sigma, x)$, which is given in Fig. 3(d). The strain values in the loading direction and the transverse direction are calculated to be $\varepsilon_{11}=-0.0157$ and $\varepsilon_{22}=0.0055$, respectively, at $1870 \mathrm{MPa}$, and the Poisson's ratio is 0.350 . These values are consistent with the macroscopic mechanical properties of this BMG. ${ }^{7}$

The parameter of $\zeta$ in Eq. (5) is a measure of the correlation length describing to what extent a correlation between a central atom and its surrounding atoms exist. In a metallic glass this is typically about $20 \AA$ and smaller in the corre- sponding liquid state. The fit results show an increase in $\zeta$ in the loading direction with stress (from 3.14 to $3.18 \AA$ ) and a decrease in the transverse direction (from 3.14 to $3.11 \AA$ ), respectively, which points to atomic rearrangements in the elastic range leading to a more ordered atomic structure in the loading direction, and a more disordered structure in the transverse direction. The reported sharpening (in the loading direction) and broadening (in the transverse direction) of the first maximum in the PDF upon loading is consistent with the observation in the parameter of $\zeta^{3}$

In summary, anisotropic atomic structural changes occur in the $\mathrm{Cu}_{64.5} \mathrm{Zr}_{35.5}$ BMG under compression in the elastic range. The atomic bond reorientations are accompanied by directionally dependent changes in the chemical and topological SRO. The MRO reflects the macroscopic strain of the BMG. Due to the atomic rearrangements upon stress, the strain values cannot be correctly determined using nearest neighbor distances.

G.W. acknowledges the Alexander von Humboldt Foundation for financial support. The authors thank S. Donath, M. Frey, and B. Opitz for technical assistance.

${ }^{1}$ S. C. Moss and J. F. Graczyk, Phys. Rev. Lett. 23, 1167 (1969); R. J. Temkin, W. Paul, and G. A. N. Connel, Adv. Phys. 22, 581 (1973).

${ }^{2}$ H. F. Poulsen, J. A. Wert, J. Neuefeind, V. Honkimäki, and M. Daymond, Nature Mater. 4, 33 (2005).

${ }^{3}$ T. C. Hufnagel, R. T. Ott, and J. Almer, Phys. Rev. B 73, 064204 (2006).

${ }^{4}$ J. Das, M. Boström, N. Mattern, A. Kvick, A. R. Yavari, A. L. Greer, and J. Eckert, Phys. Rev. B 76, 092203 (2007).

${ }^{5}$ M. Stoica, J. Das, J. Bednarcik, H. Franz, N. Mattern, W. H. Wang, and J. Eckert, J. Appl. Phys. 104, 013522 (2008)

${ }^{6}$ X. D. Wang, J. Bednarcik, H. Franz, H. B. Lou, Z. H. He, Q. P. Cao, and J. Z. Jiang, Appl. Phys. Lett. 94, 011911 (2009).

${ }^{7}$ Y. Suzuki and T. Egami, J. Non-Cryst. Solids 75, 361 (1985).

${ }^{8}$ N. Mattern, J. Bednarcik, S. Pauly, G. Wang, J. Das, and J. Eckert, Acta Mater. 57, 4133 (2009).

${ }^{9}$ W. Dmowski and T. Egami, J. Mater. Res. 22, 412 (2007).

${ }^{10}$ Y. Suzuki, J. Haimovich, and T. Egami, Phys. Rev. B 35, 2162 (1987)

${ }^{11}$ T. Egami, W. Dmowski, P. Kosmetatos, M. Boord, T. Tomida, E. Oikawa, and A. Inoue, J. Non-Cryst. Solids 192, 591 (1995).

${ }^{12}$ A. Bodapati, M. M. J. Treacy, M. Falk, J. Kieffer, and P. Keblinski, J. Non-Cryst. Solids 352, 116 (2006).

${ }^{13}$ D. Ma, A. D. Stoica, and X.-L. Wang, Nature Mater. 8, 30 (2009). 